\title{
Holistic Consideration of Patients with Schizophrenia to Improve Medication Adherence and Outcomes
}

\author{
Lan-Ting Lee ${ }^{1}$, Kao Chin Chen ${ }^{1,2}$, Wei Hung Chang ${ }^{1,3}$, Po See Chen ${ }^{1,2}$, I Hui Lee ${ }^{1,2,3}$, Yen Kuang Yang ${ }^{1,2}$ \\ ${ }^{1}$ Department of Psychiatry, National Cheng Kung University Hospital, College of Medicine, National Cheng Kung University, ${ }^{2}$ Addiction \\ Research Center, National Cheng Kung University, Tainan, ${ }^{3}$ Department of Psychiatry, National Cheng Kung University, Dou-Liou Branch, \\ Yunlin, Taiwan
}

\begin{abstract}
Although several algorithms have been applied to treat patients with schizophrenia, their clinical use remains still limited, because most emphasize the prescription of antipsychotics. A new algorithm with a more holistic approach to treating patients with schizophrenia, to be used before applying traditional prescribing guidelines, was thus proposed by an expert team of Taiwanese psychiatrists. In this algorithm, several important treatment tasks/modalities are proposed, including long-acting injection antipsychotics, shared decision-making, a case management system, compulsory treatment by law, community rehabilitation programs, the patients' feeling about their health care professionals (patients' behaviors) and their attitude/knowledge of their conditions/illness. This study proposes that evaluating the medication adherence of patients can be determined by two key domains, namely patients' behaviors and attitudes. Based on different levels of their behaviors ( $X$-axis) and attitude/knowledge ( $Y$-axis), it is possible to categorize patients with schizophrenia into six subgroups, for which various different interventions, including the use of antipsychotics, could be applied and integrated. Further research is needed to assess the applicability of this treatment algorithm in clinical settings.
\end{abstract}

KEY WORDS: Schizophrenia; Algorithms; Antipsychotics; Holistic therapies.

\section{INTRODUCTION}

Although several algorithms have been applied to treat patients with schizophrenia, their clinical use remains still limited, because most emphasize the prescription of antipsychotics. A new algorithm with a more holistic approach to treating patients with schizophrenia, to be used before applying traditional prescribing guidelines, was thus proposed by an expert team of Taiwanese psychiatrists.

\section{MAIN SUBJECTS}

\section{Antipsychotics}

Schizophrenia is a neurodegenerative disease with a high level of relapse. ${ }^{1,2)}$ The alternating nature of the ill-

\footnotetext{
Received: January 14, 2015/ Revised: March 9, 2015

Accepted: March 10, 2015

Address for correspondence: Yen Kuang Yang, MD

Department of Psychiatry, National Cheng Kung University Hospital,

138 Sheng Li Road, North Dist., Tainan 70403, Taiwan

Tel: +886-6-2353535 ext, 5213, Fax: +886-6-2084767

E-mail: ykyang@mail.ncku.edu.tw
}

ness can damage the brain, and structural abnormalities can be found even in the early phase of the disease, including ventricular enlargement and cortical atrophy. ${ }^{3-5)}$ Psychosocial and clinical impairments can become more prominent in patients with schizophrenia as the disease progresses. Therefore, it is important to provide intensive biopsychosocial interventions to improve long-term outcomes. The primary goal of treating schizophrenia is to prevent relapses and restore socio-occupational functioning to the individual's premorbid level. While it is well known that discontinuous use of antipsychotics can significantly increase the relapse rate of schizophrenia, this remains relatively high, from $41 \%$ to $97 \%$, ${ }^{6}$ particularly in the early phase of the condition, when it is approximately $53.7 \%$ to $81.9 \%{ }^{7,8)}$ The most common cause of relapse is a lack of medication adherence. ${ }^{9,10)}$ Antipsychotic discontinuation in patients with schizophrenia increases the risk of relapse by five times. ${ }^{8}$ One strategy for dealing with patients who relapse due to medication discontinuation is the use of long-acting injectable (LAI) medications, as some individuals find it easier to receive an injection at 2- to 4-week intervals than remembering to take a pill once or twice a day. ${ }^{2)}$ Indeed, several studies have demonstrated 
that LAI antipsychotics may maintain medication adherence and prevent relapse. ${ }^{11-16)}$ However, the majority of psychiatrists use LAI antipsychotics very conservatively, particularly in Asian countries. ${ }^{17,18)}$

\section{Shared Decision-making}

A related issue is how best to persuade patients to improve their medication adherence and accept LAI antipsychotics, and to this end shared decision-making (SDM) can be a very useful approach when treating individuals with chronic illnesses. ${ }^{19-21)}$ SDM aims to decrease the informational and power asymmetry between health care professionals (HCPs) and patients by increasing the latters' awareness and control regarding treatment decisions that affect their well-being. For example, one study ${ }^{1)}$ conducted an analysis of a nonrandom sample of 69 prescriber-patient conversations recorded during treatment visits. The conversations flowed freely in a number of steps, and LAI therapy was introduced with either a positive (benefits of LAIs and success stories of other patients) or a punitive approach (adherence problems and scare tactics). Patients had favorable, neutral, or resistant/concerned responses, and resistance was often related to negative feelings about injections. HCPs addressed such resistance by overcoming patient objections to LAIs (e.g., by explaining the therapeutic effects of LAIs compared to their oral counterparts) and invited patients to process of making decision. As a result, more than half $(58 \%)$ of LAI-naïve patients agreed to start LAI treatment following these office visits. If the decision was made to initiate LAI therapy, HCPs selected a specific LAI to prescribe with minimal patient input. The adherence for medication was poor in the follow-up study.

While most psychiatrists agree that SDM can lead to better outcomes than other strategies, few actually apply SDM in their clinical practice, because it is seen as time-consuming or requiring additional training. Despite these concerns, it has been demonstrated that the benefits of SDM are in fact even greater than clinicians expected before applying this approach. ${ }^{21)}$

\section{Continuity of Care and the Case Management System}

In addition to pharmacological interventions, individuals coping with schizophrenia often require a wide range of other services in order to meet their basic needs, such as those for food, shelter, and clothing. A case manager $(\mathrm{CM})$ is also needed to monitor patient drug adherence. Various types of treatment and rehabilitation programs are also required and need to be integrated in or- der for effective outcomes to be achieved. Moreover, different countries should develop culture-sensitive rehabilitation programs, because mental disorders are perceived differently in various areas. Ideally, these services should be delivered in an uninterrupted flow over time until they are able to cope or compensate for the disabilities caused by schizophrenia. Continuity of care is essential for patients with schizophrenia in all stages of the disease, but particularly for hospitalized patients in acute wards, and the main goal of continuity of care is preventing relapse. To ensure the effective integration of various types of programs it is necessary to apply a case management system and provide continuity of care. The CM is the key person in any case management system, and it is their job to examine whether patients have accepted and taken their (LAI) medications, and to work to ensure adherence if they have no being doing so. In addition, CMs also provide social support and play an important role in social resource integration to foster the independence of patients living in the community. Since there are limited resources for treating mental illness, it is important to develop a strategy that serves individuals with different needs. In other words, it is necessary to identify the best candidates for receiving CM services before patients are discharged.

\section{Compulsory Treatment by Law}

Some individuals with severe mental disorders have a lack of insight into their condition, and compulsory admission can be initiated if they are considered a danger to themselves or others. Compulsory admission allows the patient to be hospitalized for 30-60 days, depending on the related laws. In some circumstances, although the risk of suicide or homicide is significantly reduced, subjects with schizophrenia continue to show a lack of insight during their compulsory hospitalization. In some countries, discharge comes with a compulsory community treatment order to ensure that these subjects receive regular treatment in their communities. In this situation, LAI antipsychotics are the first drug of choice. For those patients with clearly lack insight into their own conditions, psychosocial interventions should be used in conjunction with medication.

\section{Designing an Algorithm before Applying Traditional Prescribing Guidelines}

Since January 2014, more than 30 Taiwanese experts have held several consensus meetings regarding treatment strategies that may lead to greater success in achieving therapeutic goals for Taiwanese patients with schizophrenia. We reached a consensus that in addition to the important 


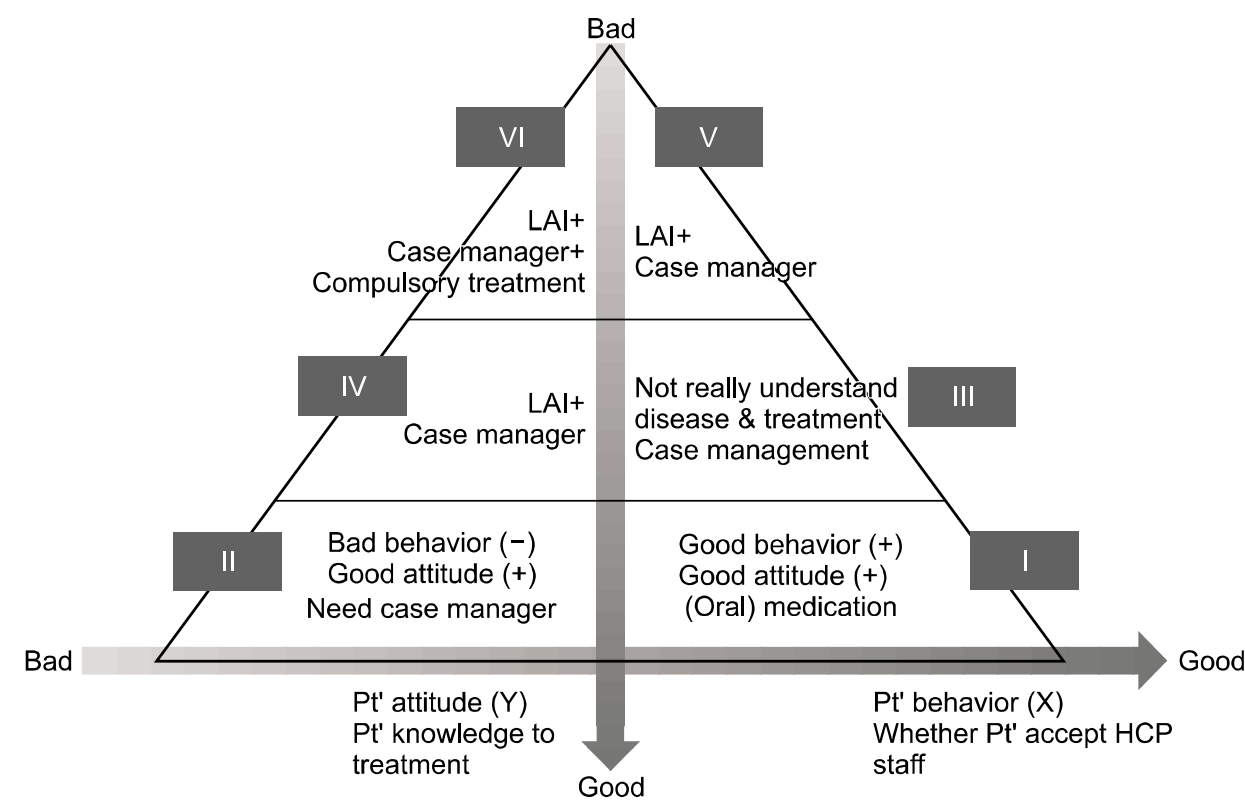

Fig. 1. New clinical practice algorithm based on patient behavior and understanding of treatment. I-VI, subgroups I-VI; LAI, longacting injectable; Pt, patient; HCP, health care professional. role of prescriber-patient interactions in SDM and patients' explanatory model (EM) for their illness, patient characteristics should be taken into account to design holistic and individualized treatment plans. Based on the literature, we developed a new algorithm (Fig. 1) that is suitable for treating patients with schizophrenia before applying traditional prescribing guidelines. ${ }^{22-27)}$ Such an algorithm could be implemented in both hospital and community-based case management settings. Medication adherence can be determined by two main dimensions: behavior (regularly visiting clinics or frequently approaching HCPs) and attitude/knowledge (a favorable response to his/her disease or understanding the nature of his/her disease). Behavior and attitude are two distinct constructs. In clinical practice, some patients may regularly "show off" in clinical settings and consent to receive LAI medication, but they have no very clear ideas about their illness. However, even patients who regularly visit clinics may not regularly take their medication. Therefore, we feel that behavioral patterns should be considered when selecting treatments and interventions (X-axis in Fig. 1). Patient attitude/knowledge (Y-axis in Fig. 1) also influences behavior, and changing/improving patients' attitude/knowledge is thus a primary goal in successful treatment programs. HCPs may start this process by assessing patients' attitudes and behavior, and then identifying appropriate intervention strategies. Patients' negative attitudes can be related to low levels of insight into their conditions, and are regarded as the primary cause of treatment non-adherence. Insight can be divided into three levels: correct and sufficient knowledge about schizophrenia (no cognitive deficit), partial insight/mild cognitive deficit (e.g., compromised memory), and absolute lack of insight (severe cognitive deficit, e.g., impaired awareness of illness or anosognosia). An anosognosia-like phenomenon is not unusual in patients with schizophrenia, and can explain why many do not take their medications and refuse any treatment, as they fail to recognize that they are ill and in need of medical care.

\section{Subgrouping Individuals with Schizophrenia Based on Behavior and Attitude}

Fig. 1 shows that the behavior and attitude of individuals in subgroup I lead them to respond favorably to a simple treatment strategy. For subgroup II, which does not take medication despite acceptance, logistic problems should be considered, and environmental interventions may improve adherence. Subgroup II's attitude/knowledge is sufficient, but their behavior toward HCPs or treatment is not always positive. For such individuals, the case management system should be initiated and combined with certain counseling techniques, such as motivation enhancement or SDM. For subgroup III, although their behavior is positive toward HCPs and treatment, cognitive deficits (e.g., impaired memory) can lead to poor adherence. In addition to assigning a CM, these patients could benefit from participating in educational programs, in which cartoon-like tools and aids are often preferable to more realistic approaches. Those patients with mild cognitive deficits and negative behavior patterns toward 


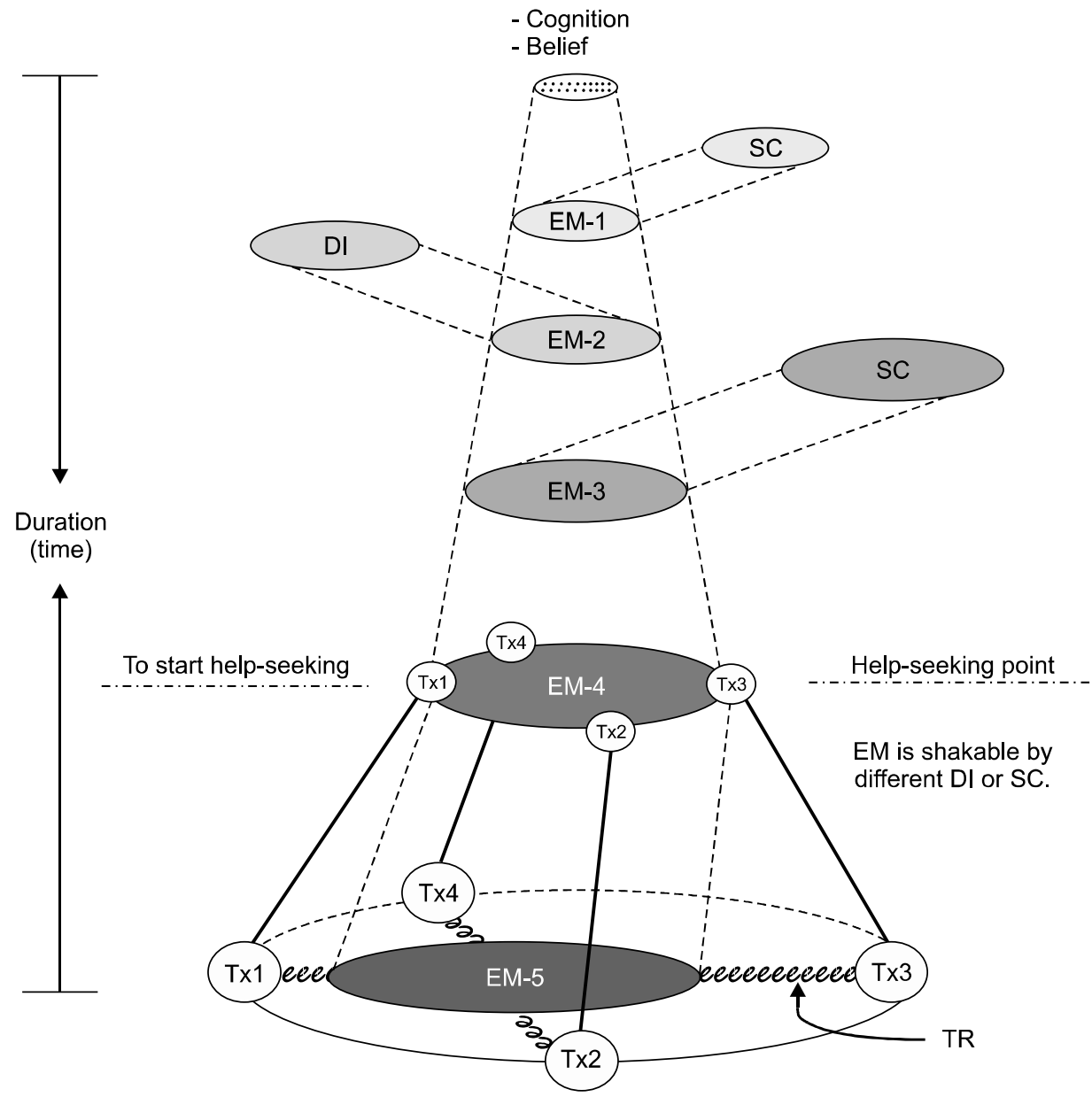

Fig. 2. The dynamic process of illness progression. Explanatory model (EM), which is composed of the various notions about condition and its treatment that are held by all those engaged in the clinical process. The EM scope was determined based on the interactions among social context, distress/ disease impact, and disease severity. The strength of adherence was determined by the treatment response to different treatment modalities. In other words, each treatment modality competes with the others. This is because people may choose to use different treatment modalities at the same time to cope as their condition progresses, particularly with regard to chronic illnesses.

DI, distress/disease impact; SC, social context/culture; Tx, treatment; TR, treatment response.

HCPs or treatment are categorized as subgroup IV. For this subset of patients, a case management system should be simultaneously considered along with LAI medication, because $\mathrm{CM}$ reminders to take oral medication are often ineffective. In subgroup $\mathrm{V}$, patients may exhibit an anosognosia-like phenomenon, even though their behavior toward treatment is positive. The strategy used with these patients is similar to that employed for subgroup IV (i.e., CM combined with LAI medication). In subgroup VI, the patient is absolutely unaware of their illness. Therefore, while these individuals have neither accepted nor started to take LAI medication, it is also very unlikely that they will accept any recommendation to do so. Even when compulsory hospitalization is needed for patients at risk of suicide or homicide, some have a persistent lack of insight after being discharged from inpatient units. In this situation, the case management system, LAI medication, and compulsory community treatment should be considered to prevent episodes in which the patients are a danger to themselves or others. Meanwhile, it is highly recom- mended that rehabilitation programs should be introduced and applied for all subgroups.

\section{Duration of Illness/Explanatory Model}

The above algorithm was proposed for individuals with schizophrenia whose caregivers or they themselves have suffered from the impact of this disease. However, in the early phase of the disease the patients and those around them might have no idea about the nature or prognosis of schizophrenia, or be reluctant to accept such a diagnosis. The patients' EM for schizophrenia is dynamic, and can influence their help-seeking behaviors. Moreover, the EM pattern might change depending on the treatment response to various different treatment modalities (Fig. 2). ${ }^{28)}$ Since the treatment response to schizophrenia is slower than that seen with many other diseases, HCPs should be more patient in working to help patients to understand and accept their illness before applying the above algorithm. 


\section{CONCLUSIONS}

The use of long-term medication treatment is a well-established response for individuals with schizophrenia. However, before applying the traditional algorithm for using antipsychotics, different modalities with regard to the EM of patients and their caregivers should be considered. HCPs thus need to understand/explore the EM and apply SDM for each patient and their caregivers before recommending any treatment modality. In this context, considering the patients' attitudes to and background knowledge of their disease and medication are essential, because, in addition to prescribing medication, combining various different modalities can be very effective for treating different patient groups. However, further research is needed to assess the applicability of this treatment algorithm in clinical settings.

\section{Acknowledgments}

The authors are indebted to the research participants, Ms. Tsai Hua Chang, Mr. Chien Ting Lin from National Cheng Kung University Hospital, and Janssen Taiwan Limited for their administrative support. The authors would also like to thank those experts who participated the consensus meetings included: Bo-Chung Chu, MD, Chao Huang, MD, Chen-Chung Liu, MD, Cheng-Chen Chang, MD, Cheng-Fang Yen, MD, Chia-Chang Chien, MD, Chien-Chi Hsu, MD, Chien-Chih Chen, MD, Chien-Liang Chen, MD, Chien-Shu Wang, MD, Ching-Yen Chen, MD, Da-Yuan Ma, MD, Fang-Huei Huang, MD, Fong-Gang Wang, MD, Hai-Ching Cheng, MD, Haw Ming-Lee, MD, Hong-Yen Lin, MD, Huang-Chi Lin, MD, Huang-Li Lin, MD, Jen-Chin Lee, MD, Kai-Da Cheng, MD, Kuan-Pin $\mathrm{Su}, \mathrm{MD}$, Li-Nen Lin, MD, Meng-Chang Tsai, MD, Min-Wei Huang, MD, Nien-Mu Chiu, MD, Peng-Wei Wang, MD, Pen-Tang Lin, MD, Philip C. Chow, MD, Ping-Yen Chen, MD, Shu-I Wu, MD, Te-Chang Changchien, MD, Tzu-Ting Chen, MD, Wei-Tsung Kao, MD, Ya-Mei Bai, MD, Yau Hung, MD, Yi-Hsing Chen, MD, Yi-Hsuan Lee, MD, and Yi-Yung Hung, MD.

\section{REFERENCES}

1. Lieberman JA. Pathophysiologic mechanisms in the pathogenesis and clinical course of schizophrenia. J Clin Psychiatry 1999;60 Suppl 12:9-12.

2. Molina V, Sanz J, Sarramea F, Benito C, Palomo T. Lower prefrontal gray matter volume in schizophrenia in chronic but not in first episode schizophrenia patients. Psychiatry Res 2004; 131:45-56.

3. Cahn W, Hulshoff Pol HE, Lems EB, van Haren NE, Schnack HG, van der Linden JA, et al. Brain volume changes in first-episode schizophrenia: a 1-year follow-up study. Arch Gen Psychiatry 2002;59:1002-1010.

4. Ho BC, Andreasen NC, Nopoulos P, Arndt S, Magnotta V, Flaum M. Progressive structural brain abnormalities and their relationship to clinical outcome: a longitudinal magnetic resonance imaging study early in schizophrenia. Arch Gen Psychiatry 2003;60:585-594.

5. Sun D, Stuart GW, Jenkinson M, Wood SJ, McGorry PD, Velakoulis D, et al. Brain surface contraction mapped in first-episode schizophrenia: a longitudinal magnetic resonance imaging study. Mol Psychiatry 2009;14:976-986.

6. Emsley R, Chiliza B, Asmal L, Harvey BH. The nature of relapse in schizophrenia. BMC Psychiatry 2013;13:50.

7. McCreadie RG, Wiles DH, Livingston MG, Watt JAG, Greene JG, Kershaw PW, et al. The Scottish first episode schizophrenia study. VIII. Five-year follow-up: Clinical and psychosocial findings. Br J Psychiatry 1992;161:496-500.

8. Robinson D, Woerner MG, Alvir JM, Bilder R, Goldman $\mathrm{R}$, Geisler S, et al. Predictors of relapse following response from a first episode of schizophrenia or schizoaffective disorder. Arch Gen Psychiatry 1999;56:241-247.

9. Weiden PJ, Olfson M. Cost of relapse in schizophrenia. Schizophr Bull 1995;21:419-429.

10. Thieda P, Beard S, Richter A, Kane J. An economic review of compliance with medication therapy in the treatment of schizophrenia. Psychiatr Serv 2003;54:508-516.

11. Zhu B, Ascher-Svanum H, Shi L, Faries D, Montgomery W, Marder SR. Time to discontinuation of depot and oral first-generation antipsychotics in the usual care of schizophrenia. Psychiatr Serv 2008;59:315-317.

12. Brnabic AJ, Kelin K, Ascher-Svanum H, Montgomery W, Kadziola Z, Karagianis J. Medication discontinuation with depot and oral antipsychotics in outpatients with schizophrenia: comparison of matched cohorts from a 12-month observational study. Int J Clin Pract 2011;65:945-953.

13. Peuskens J, Olivares JM, Pecenak J, Tuma I, Bij de Weg $\mathrm{H}$, Eriksson L, et al. Treatment retention with risperidone long-acting injection: 24-month results from the Electronic Schizophrenia Treatment Adherence Registry (e-STAR) in six countries. Curr Med Res Opin 2010;26:501-509.

14. Olivares JM, Rodriguez-Morales A, Diels J, Povey M, Jacobs A, Zhao Z, et al; e-STAR Spanish Study Group. Long-term outcomes in patients with schizophrenia treated with risperidone long-acting injection or oral antipsychotics in Spain: results from the electronic Schizophrenia Treatment Adherence Registry (e-STAR). Eur Psychiatry 2009;24:287-296.

15. Tiihonen J, Haukka J, Taylor M, Haddad PM, Patel MX, Korhonen P. A nationwide cohort study of oral and depot antipsychotics after first hospitalization for schizophrenia. Am J Psychiatry 2011;168:603-609.

16. Ren XS, Crivera C, Sikirica M, Dirani R, Qian S, Kazis LE. Evaluation of health services use following the initiation of risperidone long-acting therapy among schizophrenia patients in the veterans health administration. J Clin Pharm Ther 2011;36:383-389.

17. Heres S, Hamann J, Kissling W, Leucht S. Attitudes of psychiatrists toward antipsychotic depot medication. J Clin Psychiatry 2006;67:1948-1953.

18. Patel MX, Haddad PM, Chaudhry IB, McLoughlin S, Husain N, David AS. Psychiatrists' use, knowledge and attitudes to first- and second-generation antipsychotic long-acting injections: comparisons over 5 years. J Psychopharmacol 2010;24:1473-1482.

19. Charles C, Gafni A, Whelan T. Shared decision-making in 
the medical encounter: what does it mean? (or it takes at least two to tango). Soc Sci Med 1997;44:681-692.

20. Hamann J, Leucht S, Kissling W. Shared decision making in psychiatry. Acta Psychiatr Scand 2003;107:403-409.

21. Hamann J, Cohen R, Leucht S, Busch R, Kissling W. Shared decision making and long-term outcome in schizophrenia treatment. J Clin Psychiatry 2007;68:992-997.

22. Lehman AF, Lieberman JA, Dixon LB, McGlashan TH, Miller AL, Perkins DO, et al; American Psychiatric Association; Steering Committee on Practice Guidelines. Practice guideline for the treatment of patients with schizophrenia, second edition. Am J Psychiatry 2004;161(2 Suppl): $1-56$.

23. Hasan A, Falkai P, Wobrock T, Lieberman J, Glenthoj B, Gattaz WF, et al; World Federation of Societies of Biological Psychiatry (WFSBP) Task Force on Treatment Guidelines for Schizophrenia. World Federation of Societies of Biological Psychiatry (WFSBP) Guidelines for Biological Treatment of Schizophrenia, part 1: update 2012 on the acute treatment of schizophrenia and the management of treatment resistance. World J Biol Psychiatry 2012;13:318-378.

24. Hasan A, Falkai P, Wobrock T, Lieberman J, Glenthoj B, Gattaz WF, et al; WFSBP Task force on Treatment
Guidelines for Schizophrenia. World Federation of Societies of Biological Psychiatry (WFSBP) guidelines for biological treatment of schizophrenia, part 2: update 2012 on the long-term treatment of schizophrenia and management of antipsychotic-induced side effects. World J Biol Psychiatry 2013;14:2-44.

25. Argo TR, Crismon ML, Miller AL, Moore TA, Bendele SD, Suehs B. Texas medication algorithm project procedural manual: Schizophrenia treatment algorithms. Texas:Texas Department of State Health Services;2008.

26. Taylor D, Paton C, Kapur S. The Maudsley prescribing guidelines. 10th ed. London:Informa Healthcare;2009.

27. Kuipers E, Kendall T, Udechuku AY, Slade E, Birchwood $\mathrm{M}$, Brabban A, et al. Psychosis and schizophrenia in adults-The NICE guideline on treatment and management. National Collaborating Centre for Mental Health, commissioned by the National Institute for Health and Care Excellence; 2014.

28. Chen PS, Cheng SH, Yang YK, Yeh TL, Chen SJ, Yang MJ. Help-seeking behaviors and disease perception in psychiatric patients and their families in a teaching hospital. Chin J Fam Med (Taipei) 2002;12:32-42. 